\section{O anacronismo dos modelos assistenciais para os idosos na área da saúde: desafios para o setor privado}

\author{
Anachronic health care models for the elderly: \\ challenges for the private sector
}

\begin{abstract}
Brazil's population is aging rapidly. The average life expectancy is increasing such that a large proportion of the current population will reach old age. However, the large Brazilian cities still lack the kind of health services infrastructure needed to meet the demands resulting from present demographic changes. This article examines access to and utilization of health care among the elderly, with particular emphasis on the private sector, drawing on data from the $\mathrm{Na}$ tional Household Sample Survey (PNAD, 1998 and 2003). Socio-demographic variables included gender, age, region of residence, and family income. According to the PNAD data presented in the current study, from 1998 to 2003, health conditions improved among Brazilians aged 60 years or older.
\end{abstract}

Demographic Aging; Aged; Health Planning; Health Services Research
Renato Veras 1,2

Maria Isabel Parahyba 3

\section{Introdução}

O tema "idoso" tem aparecido constantemente na mídia brasileira. O motivo desse espaço é a expressiva ampliação da população idosa e a conseqüente transformação que se opera no interior da sociedade. Uma das grandes conquistas do século XX, a longevidade, é um fenômeno mundial e, juntamente com a queda da fecundidade, ocasiona um drástico envelhecimento na população do planeta. Este processo começou em épocas distintas, em países diferentes, e evolui em proporções variadas. No Brasil, os efeitos são ainda maiores em razão do pequeno período de tempo em que vem ocorrendo.

O Brasil envelhece rapidamente. A expectativa média de vida se amplia de tal forma que grande parte da população atual irá alcançar a velhice. Hoje existem aproximadamente 18 milhões de idosos no Brasil, o equivalente a $10 \%$ de toda a população. Estima-se que em 17 anos esse número aumentará para 33 milhões, correspondendo à previsão da população com mais de 70 anos em 2050. Embora já apresentem um perfil demográfico semelhante ao dos países do Primeiro Mundo, os grandes centros populacionais brasileiros ainda não dispõem de uma infra-estrutura de serviços que dê conta das demandas decorrentes das transformações demográficas vigentes 1 .

Além das modificações populacionais, o Brasil tem experimentado uma transição epidemiológica, com alterações relevantes no quadro de 
morbi-mortalidade. As doenças infectocontagiosas que representavam $40 \%$ das mortes registradas no país em 1950, hoje são responsáveis por menos de $10 \%$. O oposto ocorreu em relação às doenças cardiovasculares: em 1950, eram causa de $12 \%$ das mortes e, atualmente, representam mais de $40 \%$. Em menos de 40 anos, o Brasil passou de um perfil de mortalidade típico de uma população jovem para um desenho caracterizado por enfermidades complexas e mais onerosas, próprias das faixas etárias mais avançadas 2 .

Para Palloni \& Peláez ${ }^{3}$, de todas as dimensões do envelhecimento populacional, as repercussões no estado de saúde do indivíduo se afiguram como as de maior impacto no âmbito social. Desse modo, urge o desenvolvimento de propostas que rompam com o enfoque tradicional centrado no tratamento das doenças, e priorizem uma abordagem preventiva com programas abrangentes de educação, com a integralidade do cuidado hierarquizado em níveis de complexidade.

Vilar 4 estima que, grosso modo, cerca de 42 bilhões de dólares eram gastos com saúde no Brasil em 2000 e, deste total, metade era consumida pelo setor público, por meio dos governos federais, estaduais e municipais. Tais números, apesar de imprecisos, mostram um cenário bastante desigual, pois cerca de apenas $20 \%$ da população possui seguro privado do setor saúde, mas consome $50 \%$ do montante disponível de recursos de saúde. Como não houve alterações significativas nesses números, verifica-se a disparidade de recursos do setor público na comparação com o setor privado. Estes dados expressam uma grande heterogeneidade no acesso e no consumo de recursos do setor privado, quando comparado com o público. Este fato indica a necessidade de se conhecer a distribuição da oferta e demanda dos serviços de saúde.

A questão da organização do sistema para uma eficiente atenção à população idosa é mais um dos desafios que o segmento da medicina suplementar está tendo de enfrentar 5. A Agência Nacional de Saúde Suplementar (ANS) vem demonstrando crescente preocupação com esta parcela da população que, embora numericamente signifique pouco mais de $10 \%$ da população brasileira, representa um terço dos gastos de saúde; custo que tende a se ampliar, ao que tudo indica, se nada for feito 6 .

O propósito deste texto - que se baseia nos dados obtidos dos suplementos de saúde da Pesquisa Nacional por Amostra de Domicílios (PNAD), de 1998 e de 2003, realizada pelo Instituto Brasileiro de Geografia e Estatística (IBGE) e, também naqueles disponibilizados pela ANS TabNet, ferramenta que utiliza como fonte de informações o Sistema de Informações sobre Bene- ficiários (SIB/ANS) - é o de verificar o padrão de acesso e da utilização dos serviços de saúde pelo grupo etário dos idosos, com particular ênfase no setor privado.

\section{Metodologia}

Na pesquisa do IBGE de 1998 e de 2003, os dados coletados são representativos da população total residente no país, em setembro de 1998 e de 2003 - excluindo a área rural da Região Norte. A PNAD é realizada por meio de amostra probabilística de domicílios obtida em três estágios de seleção: unidades primárias (municípios), unidades secundárias (setores censitários) e unidades terciárias (unidades domiciliares), compreendendo domicílios particulares e unidades de habitação em domicílios coletivos. A amostra final foi desenhada para ser representativa da população brasileira 7 .

Do total de domicílios amostrados, perto de $81 \%$ foram entrevistados e, aproximadamente, 30 mil pessoas de 60 anos ou mais foram incluídas em cada amostra. Quando possível, todas as pessoas nos domicílios foram entrevistadas. As pessoas em domicílios coletivos foram incluídas na amostra, mas elas representavam $0,1 \%$ do total de idosos.

O levantamento realizado pela PNAD em 1998 e em 2003 abrange os temas relacionados às características dos domicílios e às características demográficas, educacionais, de trabalho e de saúde dos moradores destes domicílios. O tema Saúde é composto pelas partes características de saúde dos moradores e características de mobilidade fisica dos moradores de 14 anos ou mais de idade, e tem como objetivo a obtenção de informações que permitem delinear:

- Condições de morbidade, avaliadas por meio de restrições de atividades; auto-avaliação da situação de saúde, limitação de atividades e existência de doenças crônicas;

- Cobertura por diferentes modalidades de planos de seguro-saúde; dimensionamento da população segurada e caracterização dos planos de seguro-saúde;

- Procura de atendimento de saúde e o tipo de atendimento desejado, bem como captar alguma indicação das condições de acesso a serviços de saúde e medicamentos pela população: caracterização da demanda potencial e dos determinantes da demanda reprimida dos serviços de saúde;

- Utilização dos serviços de saúde pela população: uso de serviços de saúde por motivo, tipo de serviço, tipo de prestador, financiador e nível de satisfação dos usuários dos serviços de saúde, nos âmbitos ambulatorial e hospitalar; $\mathrm{e}$ 
- Gasto privado em saúde: gasto individual com planos de saúde, consumo de bens e serviços de saúde, incluindo gastos com consumo de medicamentos 8 .

O questionário da PNAD incluiu questões sobre cobertura de plano de saúde para todas as pessoas investigadas na amostra. As questões propostas foram as seguintes: existência de plano de saúde, plano de saúde público ou privado, avaliação do plano de saúde, titularidade, pagamento de mensalidade e amplitude do plano de saúde.

As medidas de status sócio-demográfico incluíram sexo, idade, região de residência e renda familiar. Os microdados da PNAD de 1998 e 2003 foram processados e analisados por meio do Banco Multidimensional Estatístico (IBGE/BME). A análise estatística utilizou as informações expandidas da amostra, que são representativas para o total da população idosa no Brasil.

Segundo a definição do IBGE, o BME é uma base de dados formada por microdados, originados nas pesquisas estatísticas efetuadas pelo IBGE e pela metainformação associada à sua produção. Os microdados correspondem aos dados existentes nos questionários das pesquisas. Cada questionário vai constituir um ou mais registros de informações e, portanto, compor a informação mais desagregada possível para a pesquisa estatística. Para facilitar o manuseio dos microdados são acrescentadas outras variáveis derivadas. É um instrumento particularmente útil para elaboração de análises descritivas de informações estatísticas, como o presente estudo, e está disponível na Internet para usuários cadastrados no IBGE (http:/ /www.bme.ibge.gov.br).

\section{Resultados}

Segundo as informações da PNAD de 2003, a abrangência dos planos de saúde privados entre os idosos no Brasil é de, aproximadamente, 5 milhões de brasileiros que são cobertos pelo setor da medicina suplementar, representando $29,4 \%$ do total de idosos; e em torno de 1,2 milhão possuía uma cobertura de instituto ou instituição patronal de assistência ao servidor público civil e militar.

A comparação entre os anos de 1998 e de 2003 mostra que houve aumento na proporção de idosos com plano de saúde privado, de $26,9 \%$ para $29,4 \%$, e que este acréscimo ocorreu entre os idosos com plano de empresa privada (Figura 1).

Considerando-se a cobertura por faixa etária, no setor privado, o menor percentual é observado entre as crianças $-14,2 \%$ na faixa de 0

Figura 1

População idosa (60 anos ou mais), por tipo de cobertura de plano de saúde. Pesquisa Nacional por Amostra de Domicílios, 1998 e 2003, Brasil.

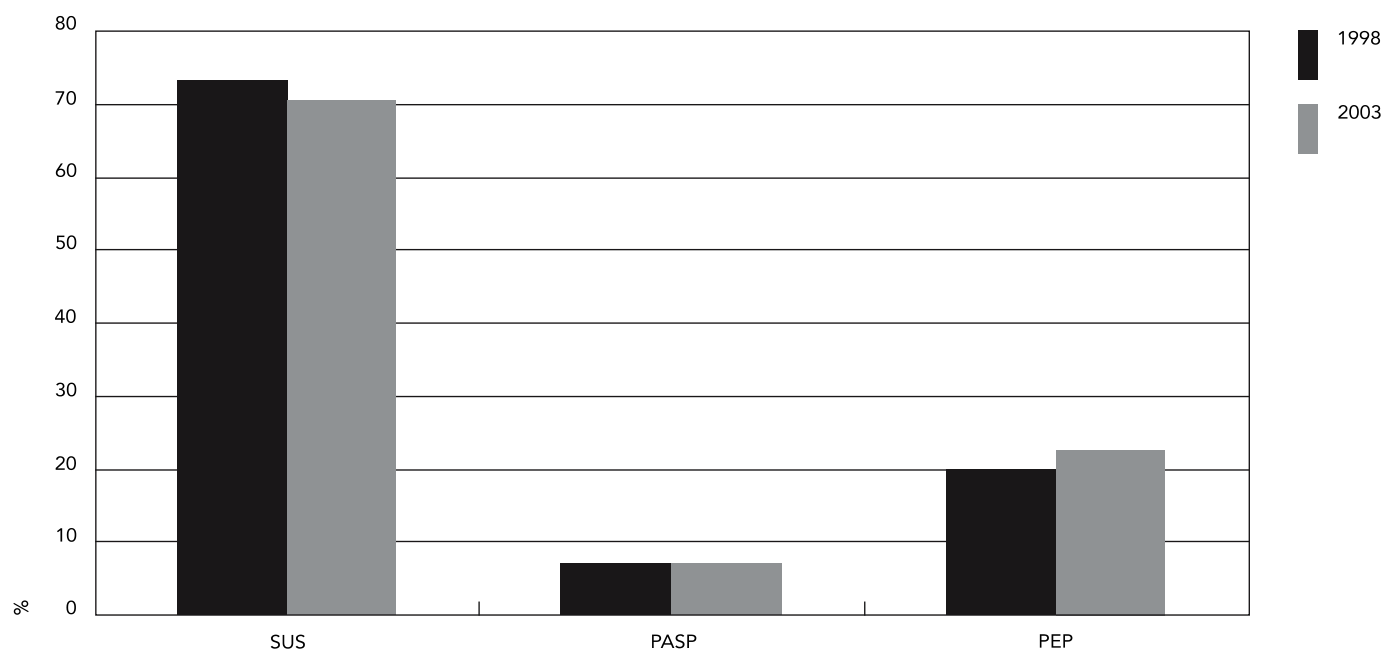

SUS: Sistema Único de Saúde; PASP: Plano de Assistência ao Servidor Público; PEP: Plano de Empresa Privada. Fonte: Pesquisa Nacional por Amostra de Domicílios, 1998 e 2003 (Instituto Brasileiro de Geografia e Estatística). 
a 19 anos -, elevando-se durante o período de vida economicamente ativa $(27,1 \%$ entre 50 e 59 anos), decaindo ao final desta (23,5\% entre $60 \mathrm{e}$ 69 anos) e voltando a crescer nas faixas etárias de idosos. As maiores coberturas são observadas nas faixas etárias de 70 a 79 anos $(26,7 \%)$ e 80 anos e mais $(30,2)$ (Figura 2).

Conforme pode ser observado na Tabela 1 , há uma associação positiva entre cobertura de plano de saúde e renda familiar. A clientela dos planos privados de saúde é predominantemente composta de pessoas de renda mais alta, ocorrendo o inverso entre os idosos que possuem apenas cobertura pelo Sistema Único de Saúde (SUS). Em 2003, entre os idosos usuários do SUS, apenas $5,8 \%$ deles apresentavam um rendimento familiar de mais de três salários mínimos per capita, enquanto entre os idosos que possuíam planos privados esta proporção alcançava $42,8 \%$. No entanto, na comparação entre 1998 e 2003, entre os mais ricos, observa-se que houve uma redução em 2003 (42,8\%) em relação a 1998 (54,8\%) entre aqueles idosos que possuíam planos de saúde.

Na Tabela 2 é demonstrado que houve uma evolução positiva do número de atendimentos à saúde entre os anos de 1998 e de 2003, quer pelo SUS, quer pelas empresas privadas prestadoras de serviços de saúde. Mesmo considerando a ampliação do número de idosos neste período, houve um aumento do número de atendimentos de saúde. Ampliação de cobertura pode ser entendida como melhoria no cuidado com os idosos.

\section{Tabela 1}

Proporção de pessoas de 60 anos ou mais de idade por cobertura de plano de saúde e rendimento mensal domiciliar em classes de salário mínimo per capita.

\begin{tabular}{lcc}
\hline & 1998 & $\mathbf{2 0 0 3}$ \\
\hline $\begin{array}{l}\text { Não tem plano de saúde } \\
<1 / 4\end{array}$ & 3,5 & \\
$1 / 4-1 / 2$ & 14,6 & 15,4 \\
$1 / 2-1$ & 36,7 & 40,5 \\
$1-3$ & 36,1 & 34,2 \\
$>3$ & 9,1 & 5,8 \\
Tem plano de saúde & & \\
$<1 / 4$ & 0,4 & 0,5 \\
$1 / 4-1 / 2$ & 1,9 & 2,2 \\
$1 / 2-1$ & 7,7 & 12,0 \\
$1-3$ & 35,2 & 42,4 \\
$>3$ & 54,8 & 42,8 \\
\hline
\end{tabular}

Fonte: Pesquisa Nacional por Amostra de Domicílios, 1998 e 2003 (Instituto Brasileiro de Geografia e Estatística).

Figura 2

Cobertura de planos de assistência médica por faixa etária. Brasil, São Paulo e Rio de Janeiro, junho de 2006.



Fonte: Sistema de Informações de Beneficiários, dezembro de 2006 (Agência Nacional de Saúde Suplementar/Ministério da Saúde). 
Na Figura 3 é ratificada a avaliação positiva dos usuários pelo Sistema Público de Saúde, já verificado em 1998, com os valores percentuais da satisfação dos usuários, nos itens "muito bom" e "bom", ampliando-se de $81.2 \%$ para $83.9 \%$ entre as duas pesquisas da PNAD.

Tais constatações reforçam a necessidade de uma análise mais cuidadosa nos dados oferecidos pelo estudo do IBGE, particularmente para a população idosa, por ser o grupo etário que mais cresce e que dele muito pouco se conhece.

Na Figura 4 é apresentada a proporção da população que, nas duas semanas anteriores à pesquisa, relatou restrição das atividades habituais em decorrência de problema de saúde. Pode-se perceber que a proporção da população que se declarou com doença não é grande, mas bem superior entre os idosos.

Na Figura 5, trabalhamos apenas com o grupo que relatou restrição de atividades e se pode verificar que quase a metade $(41,7 \%)$ é composta de idosos. Todavia, comparando-se com o ano de 1998, observa-se uma redução na proporção de pessoas de 60 anos ou mais que declararam restrição de atividades por conta de problema de saúde, de 59,9\%, em 1998, para 41,7\%, em 2003.

Com relação às doenças crônicas, em 2003, $29,9 \%$ da população brasileira declararam serem portadores de, pelo menos, uma doença crônica.

Tabela 2

Pessoas de 60 anos ou mais que tiveram atendimento de saúde nas duas últimas semanas Pesquisa Nacional por Amostra de Domicílios, 1998 e 2003, Brasil.

1998

\begin{tabular}{lcc}
\hline Atendimento por meio de plano de saúde & & \\
Tem & 836.079 & 1.123 .906 \\
Não tem & 2.095 .508 & 2.915 .115 \\
Sem declaração & 25.464 & 49.716 \\
Natureza do serviço de saúde & & \\
Público & 1.606 .606 & 2.411 .772 \\
Particular & 1.308 .392 & 1.615 .993 \\
Sem declaração & 42.053 & 60.972 \\
Total & 2.957 .051 & 4.088 .737
\end{tabular}

2003

Nota: os atendimentos se referem às doenças, aos exames de rotina, ao acidente ou à lesão, ao pré-natal, ao parto, ao tratamento médico ou à reabilitação, ao problema odontológico ou ao somente atestado.

Fonte: Pesquisa Nacional por Amostra de Domicílios, 1998 e 2003 (Instituto Brasileiro de Geografia e Estatística).

O fato marcante em relação às doenças crônicas é que elas crescem de forma muito importante com o passar dos anos. Como pode ser observado na Tabela 3, enquanto no grupo de idade compreendida entre 0 a 14 anos foram repor-

Figura 3

Avaliação do atendimento público dos idosos usuários do Sistema Único de Saúde (SUS). Pesquisa Nacional por Amostra de Domicílios, 1998 e 2003, Brasil.

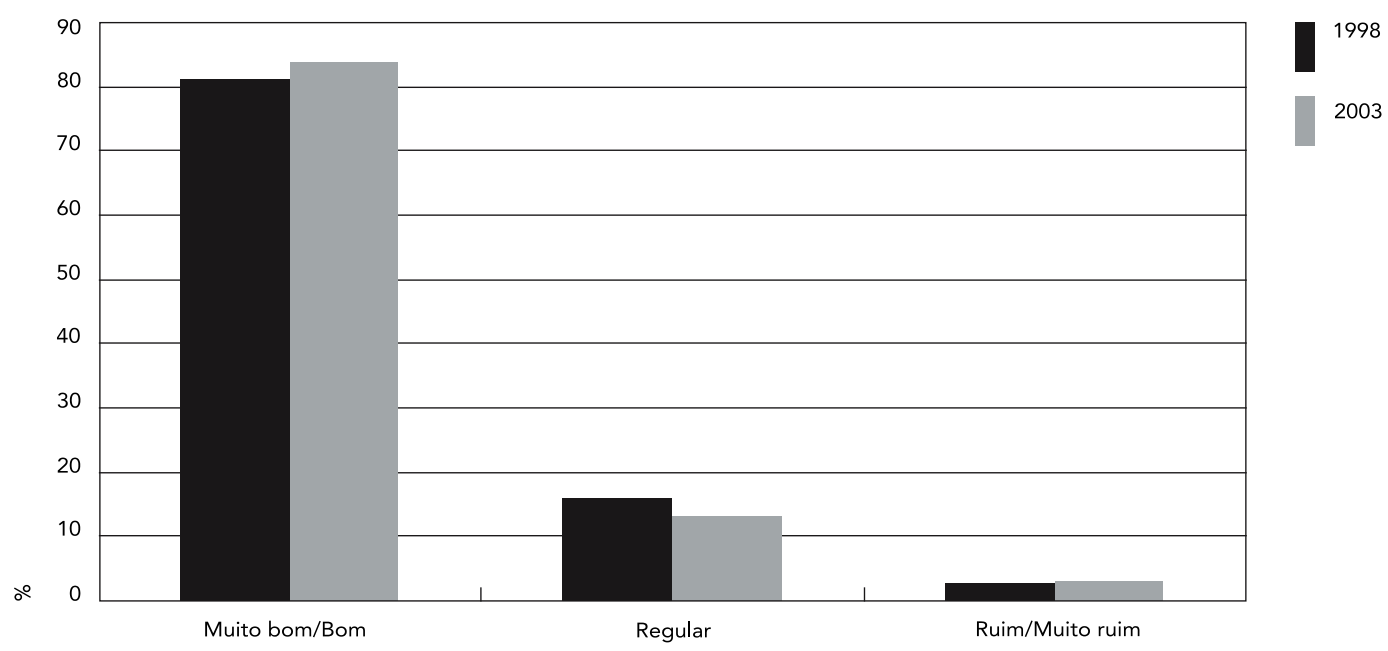

Fonte: Pesquisa Nacional por Amostra de Domicílios, 1998 e 2003 (Instituto Brasileiro de Geografia e Estatística). 
Proporção de população que, nas duas semanas precedentes à entrevista, relatou restrição de atividade em razão de problemas de saúde. Pesquisa Nacional por Amostra de Domicílios, 1998 e 2003, Brasil.

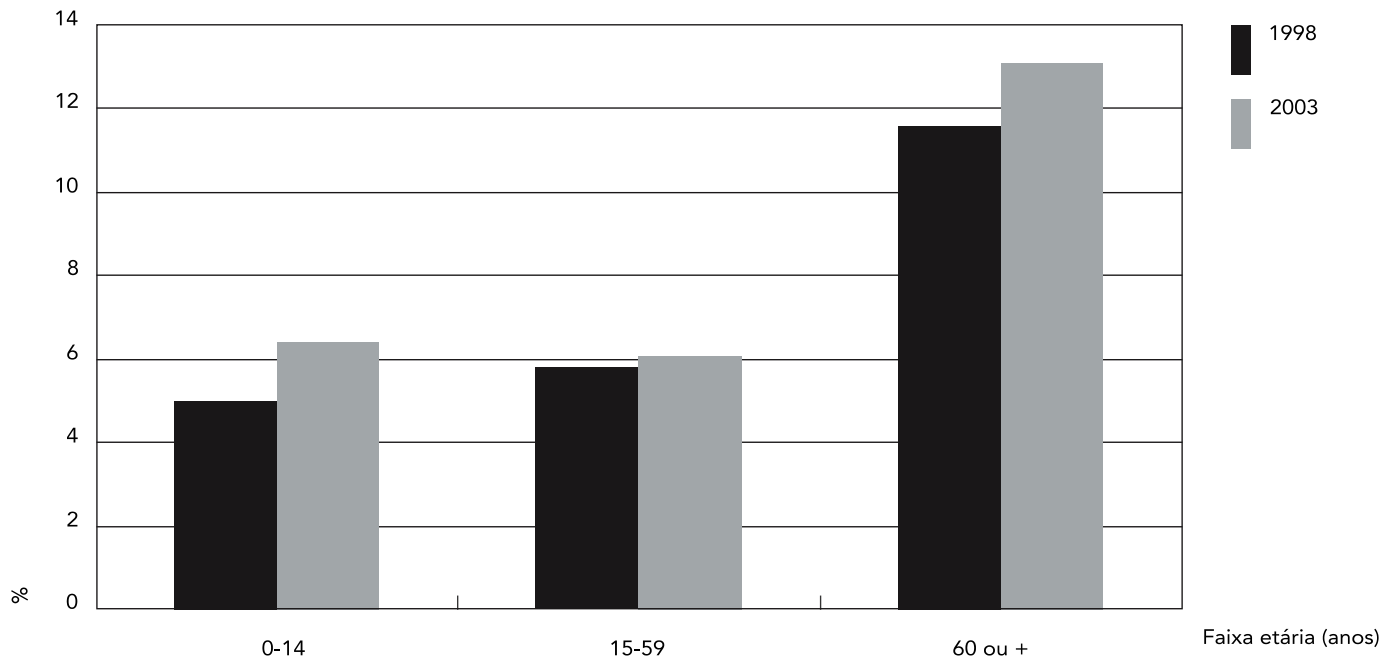

Fonte: Pesquisa Nacional por Amostra de Domicílios, 1998 e 2003 (Instituto Brasileiro de Geografia e Estatística).

Figura 5

Proporção de população com restrição de atividades por motivo de doença nas duas últimas semanas, segundo os grupos de idade. Pesquisa Nacional por Amostra de Domicílios, 1998 e 2003, Brasil.

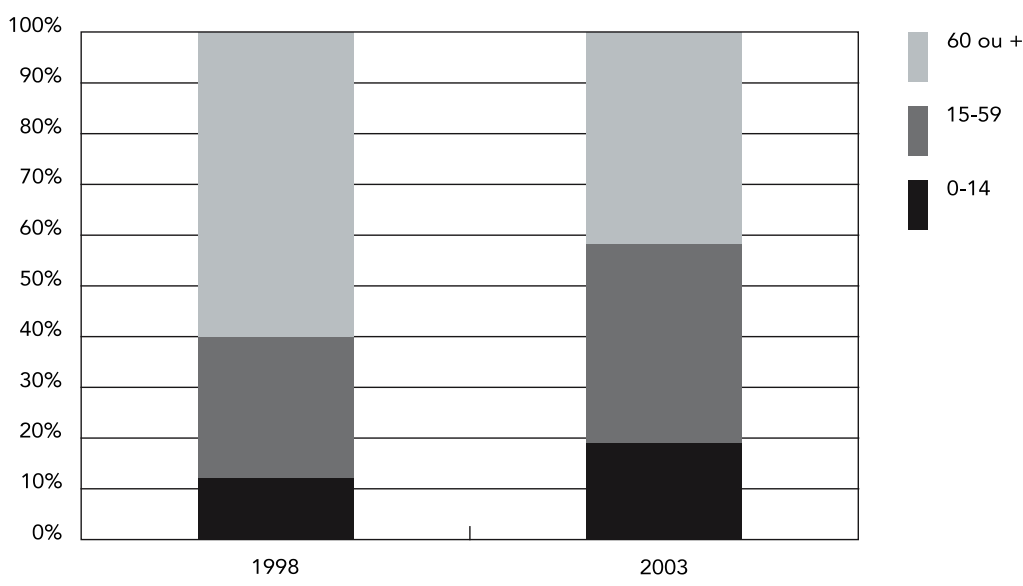

Fonte: Pesquisa Nacional por Amostra de Domicílios, 1998 e 2003 (Instituto Brasileiro de Geografia e Estatística). tados apenas 9,3\% de doenças crônicas, no dos idosos este valor atingiu $75,5 \%$.

Embora a proporção de idosos que declaram doença crônica tenha diminuído, passando de 78,7\% em 1998 para 75,5\% em 2003, a caracterís tica de múltiplas patologias entre os idosos, fica evidenciada, conforme pode ser constatado na Figura 6.

Na Figura 6 são apresentados os percentuais de pessoas que realizaram consultas médicas nos últimos 12 meses, por grupos de idade. Como é de se esperar, o grupo etário de mais de 60 anos foi o mais expressivo.

Como a população envelhece e os idosos possuem mais doenças crônicas, o número de consultas se amplia. Sabemos que mais consultas levam ao maior consumo de medicamentos, mais realização de exames complementares e mais hospitalização. As necessidades em saúde têm um padrão de distribuição segundo a idade em "J", ou seja, as pessoas no início e, sobretudo, no final da vida apresentam mais problemas de saúde (Figura 7). A grande diferença é que as doenças da faixa jovem são agudas e, portanto, de custo menor, enquanto as dos idosos são crônicas e de alto custo. A comparação das informações da PNAD para 1998 e 2003 mostra que houve acréscimo na proporção de pessoas que realizaram consulta médica em todos os grupos etários, mas 
Números absolutos e percentuais de doenças crônicas por grupo de idade. Pesquisa Nacional por Amostra de Domicílios, 1998 e 2003, Brasil.

\begin{tabular}{|c|c|c|c|c|c|c|}
\hline \multirow[t]{2}{*}{ Grupos de idade (anos) } & \multicolumn{2}{|c|}{ População } & \multicolumn{2}{|c|}{ Sem doença crônica } & \multicolumn{2}{|c|}{ Com doença crônica } \\
\hline & $\mathrm{n}$ & $\%$ & $\mathrm{n}$ & $\%$ & $\mathbf{n}$ & $\%$ \\
\hline \multicolumn{7}{|l|}{1998} \\
\hline $0-14$ & 44.063 .686 & 27,9 & 39.986 .310 & 90,8 & 3.973 .799 & 9,9 \\
\hline $15-59$ & 103.235 .426 & 65,2 & 64.661 .620 & 62,4 & 38.472 .948 & 37,3 \\
\hline 60 ou + & 13.896 .177 & 6,9 & 3.345 .289 & 30,6 & 10.931 .907 & 78,7 \\
\hline Total & 161.195 .289 & 100,0 & 107.993 .219 & 68,3 & 53.378 .654 & 31,6 \\
\hline \multicolumn{7}{|l|}{2003} \\
\hline $0-14$ & 47.793 .185 & 27,2 & 43.348 .604 & 90,7 & 4.444 .581 & 9,3 \\
\hline $15-59$ & 111.182 .801 & 63,2 & 75.780 .186 & 68,2 & 35.402 .615 & 31,8 \\
\hline $60 \mathrm{ou}+$ & 16.912 .925 & 9,6 & 4.146 .639 & 24,5 & 12.766 .286 & 75,5 \\
\hline Total & 175.888 .911 & 100,0 & 123.275 .429 & 70,1 & 52.613 .482 & 29,9 \\
\hline
\end{tabular}

Fonte: Pesquisa Nacional por Amostra de Domicílios, 1998 e 2003 (Instituto Brasileiro de Geografia e Estatística).

especialmente entre os idosos, (72,2\%, em 1998, e 78\%, em 2003).

$\mathrm{O}$ aumento do número médio de consultas médicas realizadas nos últimos 12 meses anteriores à data da pesquisa confirma a maior procura de atendimento por parte da população, mormente a idosa. A Figura 8 mostra que, em 1998, o número médio, entre os idosos, era de 3,7 consultas, aumentando para 4,3 em 2003.

\section{Discussão}

Estes dados devem permitir que os responsáveis pela área da saúde reflitam sobre o impacto deste grupo etário na organização dos serviços de saúde.

A mudança no perfil epidemiológico acarreta crescimento das despesas com tratamentos médicos e hospitalares, ao mesmo tempo em que se configura em desafio para as autoridades sanitárias, em especial no tocante à implantação de novos modelos e métodos para o enfrentamento do problema. O idoso consome mais serviços de saúde, as internações hospitalares são mais freqüentes e o tempo de ocupação do leito é maior em virtude da multiplicidade de suas patologias, quando comparado às de outras faixas etárias 9 .

Os dados coletados pela PNAD mostram que os planos de saúde atuam no sistema brasileiro de saúde introduzindo um elemento de desigualdade social no acesso e na utilização dos serviços de saúde, porque cobrem majoritariamente o segmento populacional de maior renda. Apesar das evidências que demonstram que os pacientes do SUS são menos saudáveis do que os
Figura 6

Proporção de pessoas de 60 anos ou mais que declararam ter doença crônica, segundo o número de doenças. Pesquisa Nacional por Amostra de Domicílios, 1998 e 2003, Brasil.

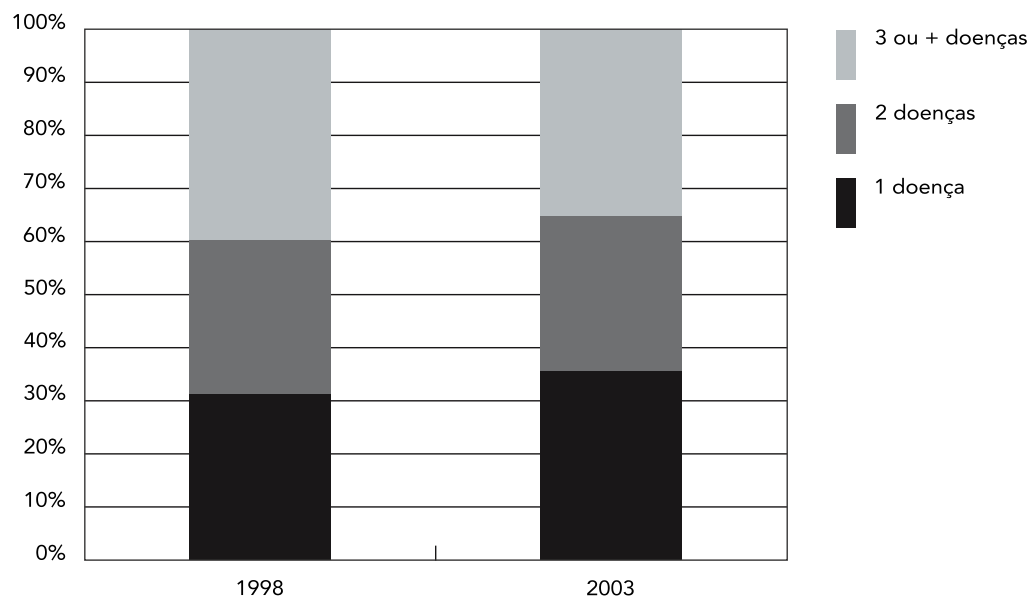

Fonte: Pesquisa Nacional por Amostra de Domicílios, 1998 e 2003 (Instituto Brasileiro de Geografia e Estatística).

associados das prestadoras de saúde, a proporção de atendimentos é superior no setor privado, quando comparado com o público. Na pesquisa da PNAD e em vários estudos se ratifica a associação inversa entre saúde e pobreza 9 .

A ampliação do número de idosos e a maior utilização do sistema de saúde, conseqüência do maior tempo de vida e das múltiplas patologias 
Figura 7

Proporção de pessoas que realizaram consultas nos últimos 12 meses, segundo os grupos de idade. Pesquisa Nacional por Amostra de Domicílios, 1998 e 2003, Brasil.

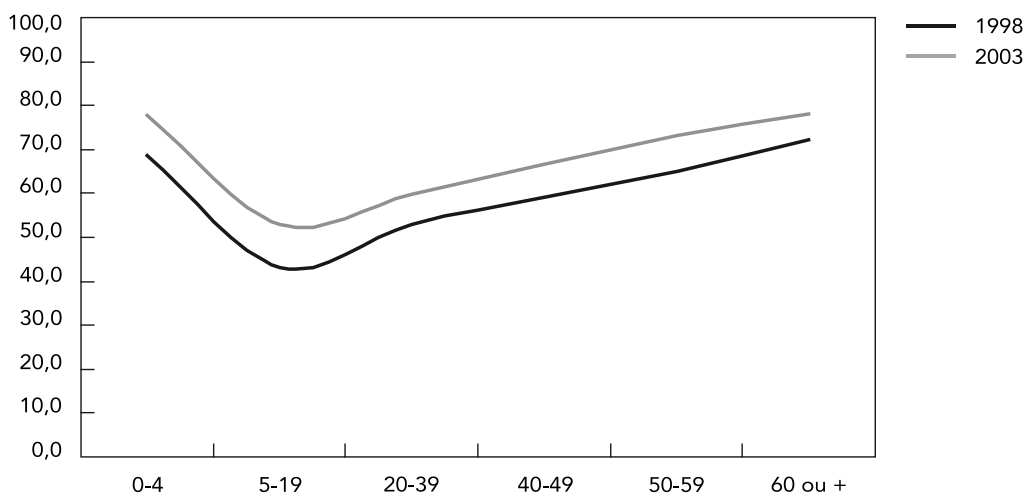

Fonte: Pesquisa Nacional por Amostra de Domicílios, 1998 e 2003 (Instituto Brasileiro de Geografia e Estatística). saúde estão provando eficácia em todo o mundo. Estudos internacionais mais recentes confirmam essas tendências e indicam uma redução no declínio funcional entre os idosos 10,11. O cenário, por conseguinte, aponta na direção de uma população idosa mais saudável, mesmo a despeito das conseqüências que o processo de envelhecimento populacional acarreta no tocante à ampliação das doenças crônicas.

Os dados da PNAD relatados no presente estudo sobre as condições gerais de saúde da população idosa no Brasil mostram que ocorreu, entre o período de 1998 e 2003, uma melhoria das condições de saúde das pessoas de 60 anos ou mais, fato que pode parecer estranho para o leitor menos atento. As causas multifatoriais que justificam este avanço são, entre outras, a melhoria da tecnologia médica e do status sócio-econômico, as mudanças comportamentais ocorridas, a adoção de programas sociais de transferência de renda focados nos mais pobres e o aumento do nível educacional dos idosos 12 .

As pesquisas da PNAD confirmam também uma informação que desmistifica o índice de satisfação dos usuários do sistema público de saúde (SUS). Quando o idoso que utiliza o sistema público é convidado a avaliar o atendimento recebido, uma expressiva maioria aprova o serviço prestado. Apenas 2,9\% dos entrevistados, no ano

Figura 8

Número médio de consultas realizadas nos últimos 12 meses, segundo os grupos de idade. Pesquisa Nacional por Amostra de Domicílios, 1998 e 2003, Brasil.

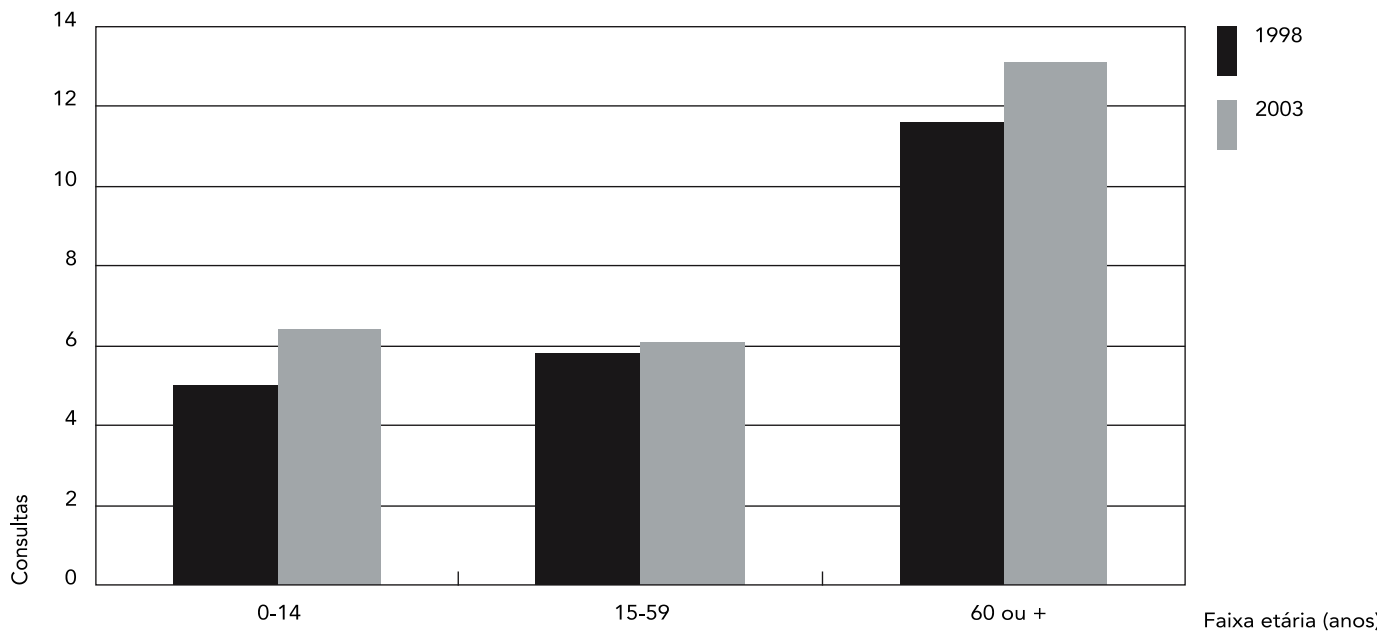

Fonte: Pesquisa Nacional por Amostra de Domicílios, 1998 e 2003 (Instituto Brasileiro de Geografia e Estatística). 
de 1998 , e $3 \%$ no de 2003 , acharam o seu funcionamento ruim ou muito ruim (Figura 3 ).

A relação entre planos privados de saúde e renda reforça a percepção por parte da população idosa de que aconteceu alguma melhoria assistencial ou, pelo menos, a ampliação de oferta de serviço por parte do SUS, pois com a ampliação de renda, a tendência natural seria o aumento de idosos filiados a planos privados de saúde. De fato houve, conforme mostra a Figura 1, a ampliação de idosos nos planos privados, porém, ao se introduzir a informação de renda, verifica-se que entre os de maior poder aquisitivo ocorreu o inverso (Tabela 1), vale dizer, uma redução de idosos, entre os mais ricos, no segmento privado de saúde. Uma hipótese a ser confirmada pela nova série da PNAD é a de verificar se é entre aqueles de maior renda, que certamente são também os de maior escolaridade, que se inicia o processo de valorização do SUS.

O número de pessoas idosas que buscou os serviços de saúde nas duas semanas anteriores à pesquisa, em 2003, foi superior a 4 milhões atendimentos, e destes, em torno de $40 \%$ possuíam cobertura através de planos de saúde. Observa-se também, na Tabela 2, a ampliação das consultas, seja no setor privado, seja no SUS, entre os anos de 1998 e 2003. O fato vem confirmar a tendência de ampliação crescente dos idosos nos sistemas de saúde, conseqüência do aumento do tempo de vida e dos anos vividos com alguma doença crônica. Esse é mais um elemento que aponta em direção à necessidade de uma gestão eficiente e diferenciada da oferecida no dias atuais.

\section{Conclusões e recomendações}

Os dados comparativos da PNAD deveriam estimular o setor privado a desenhar modelos mais contemporâneos, que oferecessem maior resolubilidade e um custo mais adequado em relação ao disponibilizado nos dias atuais 13,14. Sabendose que é grande a parcela de pessoas idosas, não fragilizadas - logo ainda com boas condições de saúde, com a maioria na idade avançada, e com renda, em média, superior ao conjunto da população -, poder-se-ia propor uma política com foco na manutenção da capacidade funcional, em programas de prevenção, no investimento de metodologias para a detecção precoce de doenças, no monitoramento das doenças crônicas, no sistema do médico personalizado, entre outras medidas, em lugar do modelo de demanda espontânea que tem no hospital a peça central do sistema.

A proposta-chave para este grupo etário é o da integralidade no cuidado, visando à postergação do início das doenças, o mais precocemente possível, com o uso dos instrumentos de identificação de risco. Desse modo, ênfase deve ser conferida à manutenção da capacidade funcional do idoso, visando buscar a compressão da morbidade, termo cunhado por Fries \& Crapo 10 , que significa desenvolver estratégias que objetivem levar a vida para o limiar mais próximo possível do limite máximo da espécie humana, mas com qualidade de vida, com autonomia e independência, isto é, com capacidade funcional.

Portanto, esses dados visam sensibilizar os tomadores de decisão para a necessidade de novas estratégias. Estabelecida a evidência do processo das transformações que se operam no setor saúde, em decorrência da transição demográfica e epidemiológica da população brasileira, foco especial deve ser dirigido ao cuidado do idoso e aos portadores de doença crônica, que são os que mais demandam por serviços de saúde. 


\section{Resumo}

O Brasil envelhece rapidamente. A expectativa média de vida se amplia de tal forma que grande parte da população atual irá alcançar a velhice. Os grandes centros populacionais brasileiros, entretanto, ainda não dispõem de uma infra-estrutura de serviços que dê conta das demandas decorrentes das transformações demográficas vigentes. O propósito deste texto é o de verificar o padrão do acesso e utilização de saúde no grupo etário dos idosos, com particular ênfase no setor privado. Foram utilizadas as informações dos Suplementos de Saúde da Pesquisa Nacional por Amostra de Domicílios (PNAD) de 1998 e 2003, realizada pelo Instituto Brasileiro de Geografia e Estatística (IBGE). As medidas de status sócio-demográfico incluíram sexo, idade, região de residência e renda familiar. Os microdados da PNAD, de 1998 e 2003, foram processados e analisados por meio do Banco Multidimensional Estatístico (BME/IBGE). Os dados da PNAD, apresentados no presente estudo, sobre as condições gerais de saúde da população idosa no Brasil, mostram que houve, entre o período de 1998 e 2003, uma melhoria das condições de saúde das pessoas de 60 anos ou mais.

Envelhecimento da População; Idoso; Planejamento em Saúde; Pesquisa sobre Serviços de Saúde

\section{Colaboradores}

R. P. Veras foi responsável pela concepção, redação e revisão do artigo. M. I. Parahyba foi responsável pela extração dos dados no BME, elaboração das tabelas, dos gráficos, redação da Metodologia e parte dos Resultados.

\section{Referências}

1. Veras RP, Parahyba MICA. A população idosa no Brasil: considerações acerca do uso de indicadores de saúde. In: Minayo MCS, organizadora. Os muitos Brasis: saúde e população na década de 80. São Paulo: Editora Hucitec/Rio de Janeiro: ABRASCO; 1994. p. 320-37.

2. Gordilho A, Sérgio J, Silvestre J, Ramos LR, AlvesFreire MP, Espindola N, et al. Desafios a serem enfrentados no terceiro milênio pelo setor saúde nas políticas de cuidado integral ao idoso. Rio de Janeiro: Universidade Aberta da Terceira da Idade/ Universidade do Estado do Rio de Janeiro; 2000.

3. Palloni A, Peláez M. SABE: survey on health and well-being of elders: preliminary report. Washington DC: Pan-American Health Organization; 2002.

4. Vilar JM. A crise no setor saúde e o ranking da OMS. Revista Brasileira de Home Care 2000; 6:22.
5. Malta DC, Jorge AO. Breve caracterização da saúde suplementar. In: Ministério da Saúde, organizador. Duas faces da mesma moeda: microrregulação e modelos assistenciais na saúde suplementar. Rio de Janeiro: Ministério da Saúde/Agência Nacional de Saúde Suplementar; 2005. p. 37-60.

6. Veras RP. Modelos contemporâneos no cuidado à saúde: novos desafios em decorrência da mudança do perfil epidemiológico da população brasileira. Revista USP 2001; (51):72-85.

7. Biachini ZM, Albieri S. A review of major household sample survey designs used in Brazil. In: Proceedings of the Joint/ASS/IAOS Conference. Aguas Calientes: International Association of Survey Statisticians; 1998. 
8. Instituto Brasileiro de Geografia e Estatística. Acesso e utilização de serviços de saúde: Pesquisa Nacional por Amostra de Domicílios (PNAD). Rio de Janeiro: Instituto Brasileiro de Geografia e Estatística; 2000.

9. Veras RP. País jovem com cabelos brancos: a saúde do idoso no Brasil. Rio de Janeiro: Editora RelumeDumará; 1994.

10. Schoeni RF, Martin LG, Andreski PM, Freedman VA. Persistent and growing socioeconomic disparities in disability among the elderly: 1982-2002. Am J Public Health 2005; 95:2065-70.
11. Fries JF, Crapo LM. Vitality and aging: implications of the rectangular curve. San Francisco: WH Freeman and Company; 1981.

12. Parahyba MI, Veras RP. Diferenciais sócio-demográficos no declínio funcional em mobilidade física entre os idosos no Brasil. Cad Saúde Pública; submetido.

13. Macera AP, Saintive MB. O mercado de saúde suplementar no Brasil. Brasília: Secretaria de Acompanhamento Econômico, Ministério da Fazenda; 2004.

14. Pinto LP, Soranz DR. Planos privados de assistência à saúde: cobertura no Brasil. Ciênc Saúde Coletiva 2004; 9:85-96.

Recebido em 07/Ago/2006

Versão final reapresentada em 04/Mai/2007

Aprovado em 29/Mai/2007 